\title{
Correction to: Subtraction improves the accuracy of coronary CT angiography for detecting obstructive disease in severely calcified segments
}

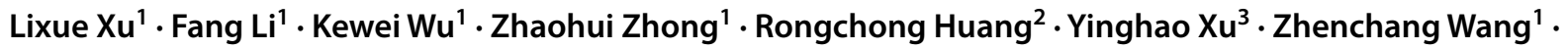 \\ Zhenghan Yang ${ }^{1} \cdot \mathrm{Yi} \mathrm{He}^{1}$
}

Published online: 16 September 2021

(c) European Society of Radiology 2021

\section{Correction to: European Radiology (2021) 31:6211-6219} https://doi.org/10.1007/s00330-021-08092-5

The original version of this article, published on 17 June 2021, unfortunately contained a mistake. The following correction has therefore been made in the original: The funding information and the conflict of interest were incorrect; the correct statements are given below. The original article has been corrected.

Funding This study has received funding from the National Key Research and Development Program of China (grant 2019YFE0107800), National Natural Science Fondation of China (grants 81671650, 81971569), and Beijing Municipal Science and technology commission (grant Z201100005620009).
Conflict of interest One of the authors of this manuscript (Yinghao Xu) is an employee of Canon Medical Systems. The remaining authors declare no relationships with any companies whose products or services may be related to the subject matter of the article.

Publisher's note Springer Nature remains neutral with regard to jurisdictional claims in published maps and institutional affiliations.

The original article can be found online at https://doi.org/10.1007/ s00330-021-08092-5.

Zhenghan Yang

yangzhenghan@vip.163.com

$\triangle \mathrm{YiHe}$

Heyi139@sina.com

1 Department of Radiology, Beijing Friendship Hospital, Capital Medical University, No. 95 YongAn Road, Beijing 100050, People's Republic of China

2 Department of Cardiology, Beijing Friendship Hospital, Capital Medical University, No. 95 YongAn Road, Beijing 100050, People's Republic of China

3 CanonMedical Systems (China) CO., LTD., Building 205, Yard NO. A10, JiuXianQiao North Road, Beijing 100015, People's Republic of China 